\title{
APPROXIMATION OF A MARTENSITIC LAMINATE WITH VARYING VOLUME FRACTIONS*
}

\author{
Bo Li $^{1}$ AND Mitchell LUSKiN ${ }^{2}$
}

\begin{abstract}
We give results for the approximation of a laminate with varying volume fractions for multi-well energy minimization problems modeling martensitic crystals that can undergo either an orthorhombic to monoclinic or a cubic to tetragonal transformation. We construct energy minimizing sequences of deformations which satisfy the corresponding boundary condition, and we establish a series of error bounds in terms of the elastic energy for the approximation of the limiting macroscopic deformation and the simply laminated microstructure. Finally, we give results for the corresponding finite element approximation of the laminate with varying volume fractions.

Résumé. Nous considérons des problèmes de minimisation d'énergie avec de multiples puits de potentiel. De tels problèmes modélisent, pour des cristaux martensitiques, des transitions de phase d'un réseau orthorhombique à monoclinique, ou d'un réseau cubique à tétragonal, par exemple.

Des résultats d'approximation des structures laminaires correspondantes, avec fractions volumiques variables, sont donnés. Des suites minimisantes, avec déformations compatibles avec les conditions au bord, sont construites et permettent l'obtention de plusieurs estimations d'erreur concernant l'approximation de la déformation macroscopique limite en fonction de l'énergie élastique.

Finalement, nous décrivons des résultats concernant l'approximation par éléments finis de la structure laminaire avec fractions volumiques variables.
\end{abstract}

AMS Subject Classification. 49M15, 65C20, 65N30, 73C50, 73K20.

Received: March 1997. Revised: January 4, 1998.

\section{INTRODUCTION}

The recently developed geometrically nonlinear theory of martensite predicts the martensitic microstructure to be the limiting configuration of energy minimizing sequences of deformations for a nonconvex energy $[2,3$, $10,14,15,18,19,21,24]$. In this theory, the energy density is minimized on multiple energy wells $\operatorname{SO}(3) U_{1} \cup$ $\cdots \cup \mathrm{SO}(3) U_{N}$ where $U_{1}, \cdots, U_{N}$ for $N>1$ are symmetry-related transformation strains representing distinct variants of the martensite and $\mathrm{SO}(3)$ is the set of all $3 \times 3$ real orthogonal matrices with determinant equal to one. Although the effect of surface energy makes a homogeneous deformation most stable, for certain boundary constraints or loading conditions the elastic energy of a martensitic crystal can be lowered as much as possible only by the fine-scale mixing of deformation gradients from distinct energy wells. A common example of such

Key words and phrases. martensitic transformation, simple laminate, volume fraction, Young measure, finite element, error estimate

* This work was supported in part by the NSF through grant DMS 95-05077, by the AFOSR through grants AF/F 49620-96-1-0057 and AF/F 49620-97-1-0187, by ARPA/URI/ONR N00014-92-J-1890, by the Institute for Mathematics and its Applications, and by a grant from the Minnesota Supercomputer Institute.

${ }^{1}$ Department of Mathematics, University of California, Los Angeles, 405 Hilgard Avenue, Los Angeles, CA 90095-1555, USA. e-mail: bli@math.ucla.edu

${ }^{2}$ School of Mathematics, University of Minnesota, 206 Church Street, S.E., Minneapolis, MN 55455, USA. e-mail: luskin@math.umn.edu 
a microstructure is a simple laminate in which the deformation gradient oscillates in parallel layers of finescale between two compatible, stress-free, homogeneous states [4,5]. More complex microstructures have been described using the notion of Young measure which gives the volume fraction for the mixing of the deformation gradients of the energy minimizing sequences of deformations $[2,3,20,32,33]$.

We focus on martensitic crystals that can undergo either an orthorhombic to monoclinic or a cubic to tetragonal transformation [3,24]. A martensitic crystal which can undergo an orthorhombic to monoclinic transformation has two symmetry-related martensitic variants $(N=2)$, and hence the elastic energy density has two wells. The more commonly observed cubic to tetragonal transformation has three symmetry-related martensitic variants $(N=3)$, so the elastic energy density has three wells. For both transformations, Ball and James have shown for boundary data which are consistent with a first-order laminate with constant volume fractions that the unique energy minimizing microstructure is the first-order laminate [3].

In this paper, we present an approximation theory for first-order laminates with varying volume fractions. We establish a series of error bounds in terms of the elastic energy of deformations for the $L^{2}$ approximation of the directional derivative of the limiting macroscopic deformation in any direction tangential to the parallel layers of the laminate, for the $L^{2}$ approximation of the limiting macroscopic deformation, for the approximation of volume fractions of the participating martensitic variants, and for the approximation of nonlinear integrals of deformation gradients.

We also give corresponding error estimates for conforming finite element approximations of the laminate with varying volume fractions. For simplicity of exposition, we restrict our analysis to continuous, piecewise linear, tetrahedral finite elements; but our analysis can be directly extended to higher order finite elements. We construct quasi-optimal finite element deformations, and we give corresponding error estimates for quasi-optimal finite element deformations.

The main framework of our analysis is the approximation theory developed for simple laminates with constant volume fractions for a two-well problem which applies to the orthorhombic to monoclinic transformation [25]. This analysis was extended to the cubic to tetragonal transformation in [22]. For constant volume fractions, an analysis for a nonconforming finite element approximation was given in [23].

A theory of numerical analysis for the microstructure in nonconvex variational problems was developed in $[12,13]$, and extended in $[7-9,17,26]$. Analyses of the approximation of relaxed variational problems have been given in $[6,16,27-29,31]$. We refer to the recent article [24] for a survey of models, computation, and numerical analysis for martensitic microstructure.

In Section 2, we describe the multi-well energy minimization problems. In Section 3, we construct energy minimizing sequences of deformations which satisfy the corresponding nonhomogeneous boundary condition. In Sections 4 and 5, we establish a series of error bounds in terms of the elastic energy of deformations for the approximation of the limiting macroscopic deformation and the approximation of the microstructure. Finally, in Section 6, we give error estimates for the approximation by quasi-optimal finite element deformations.

\section{ENERGY MINIMIZATION PROBLEMS}

In this section, we give a summary of the properties and known results for the orthorhombic to monoclinic and cubic to tetragonal martensitic transformations [2,3,24].

An orthorhombic to monoclinic transformation for a martensitic crystal is determined by its martensitic variants

$$
U_{1}=\left(I+\eta e_{1} \otimes e_{2}\right) D, \quad U_{2}=\left(I-\eta e_{1} \otimes e_{2}\right) D,
$$

where $I$ is the identity transformation from $\mathbb{R}^{3}$ to $\mathbb{R}^{3}, \eta>0$ is a material parameter, $\left\{e_{1}, e_{2}, e_{3}\right\}$ is an orthonormal basis for $\mathbb{R}^{3}$, and $D$ is a symmetric, positive definite, linear transformation from $\mathbb{R}^{3}$ to $\mathbb{R}^{3}$, given by

$$
D=d_{1} e_{1} \otimes e_{1}+d_{2} e_{2} \otimes e_{2}+d_{3} e_{3} \otimes e_{3}
$$


for some $d_{1}, d_{2}, d_{3}>0$. A cubic to tetragonal transformation for a martensitic crystal is determined by its martensitic variants

$$
\begin{gathered}
U_{1}=\eta_{1} I+\left(\eta_{2}-\eta_{1}\right) e_{1} \otimes e_{1}, \quad U_{2}=\eta_{1} I+\left(\eta_{2}-\eta_{1}\right) e_{2} \otimes e_{2} \\
U_{3}=\eta_{1} I+\left(\eta_{2}-\eta_{1}\right) e_{3} \otimes e_{3}
\end{gathered}
$$

where $\eta_{1}>0$ and $\eta_{2}>0$ are material parameters such that $\eta_{1} \neq \eta_{2}$, and $\left\{e_{1}, e_{2}, e_{3}\right\}$ is again an orthonormal basis for $\mathbb{R}^{3}$.

For a given martensitic crystal, we denote by $\Omega$ the reference configuration which is taken to be the homogeneous austenitic state at the transformation temperature. We assume that $\Omega \subset \mathbb{R}^{3}$ is a connected, bounded, open set with a Lipschitz continuous boundary. We also denote the elastic energy density of the crystal at a fixed temperature below the transformation temperature by the continuous function $\phi: \mathbb{R}^{3 \times 3} \rightarrow \mathbb{R}$, where $\mathbb{R}^{3 \times 3}$ denotes the set of all $3 \times 3$ real matrices. The elastic energy of a deformation $y: \Omega \rightarrow \mathbb{R}^{3}$ is given by

$$
\mathcal{E}(y)=\int_{\Omega} \phi(\nabla y(x)) d x
$$

where $\nabla y: \Omega \rightarrow \mathbb{R}^{3 \times 3}$ is the deformation gradient. We define the set of deformations of finite energy by

$$
W^{\phi}=\left\{y \in C\left(\bar{\Omega} ; \mathbb{R}^{3}\right): \int_{\Omega} \phi(\nabla y(x)) d x<\infty\right\} .
$$

We assume that the energy density $\phi$ is minimized on the energy wells

$$
\mathcal{U}_{i}=\mathrm{SO}(3) U_{i}, \quad i \in K,
$$

where $K=\{1,2\}$ for the orthorhombic to monoclinic transformation and $K=\{1,2,3\}$ for the cubic to tetragonal transformation. Thus, we may assume after adding a constant to the energy density that

$$
\begin{array}{cc}
\phi(F) \geq 0, \quad \forall F \in \mathbb{R}^{3 \times 3}, \\
\phi(F)=0 \quad \text { if and only if } \quad F \in \mathcal{U} \equiv \cup\left\{\mathcal{U}_{i}: i \in K\right\} .
\end{array}
$$

We shall also assume that the energy density $\phi$ grows quadratically away from the energy wells, that is,

$$
\phi(F) \geq \kappa\|F-\pi(F)\|^{2}, \quad \forall F \in \mathbb{R}^{3 \times 3},
$$

where $\kappa>0$ is a constant and $\pi: \mathbb{R}^{3 \times 3} \rightarrow \mathcal{U}$ is a Borel measurable projection defined by

$$
\|F-\pi(F)\|=\min _{G \in \mathcal{U}}\|F-G\|, \quad \forall F \in \mathbb{R}^{3 \times 3},
$$

and where

$$
\|F\|=\left(\sum_{i, j=1}^{3} F_{i j}^{2}\right)^{\frac{1}{2}}, \quad \forall F=\left(F_{i j}\right) \in \mathbb{R}^{3 \times 3} .
$$

The projection $\pi(F)$ exists for any $F \in \mathbb{R}^{3 \times 3}$, since $\mathcal{U}$ is compact, although the projection may not be unique. It is easy to see that

$$
\operatorname{det} F=d_{1} d_{2} d_{3}>0, \quad \forall F \in \mathcal{U} \equiv \cup\left\{\mathcal{U}_{i}: i \in K\right\},
$$


for the orthorhombic to monoclinic transformation, and that

$$
\operatorname{det} F=\eta_{1}^{2} \eta_{2}>0, \quad \forall F \in \mathcal{U} \equiv \cup\left\{\mathcal{U}_{i}: i \in K\right\},
$$

for the cubic to tetragonal transformation.

We note that every function in $W^{1, \infty}(\Omega)$ is equal almost everywhere to a unique Lipschitz continuous function defined on $\bar{\Omega}[1]$. For simplicity in what follows, we shall consider only the Lipschitz continuous representatives of functions in $W^{1, \infty}(\Omega)$. Thus, we shall consider functions in $W^{1, \infty}(\Omega)$ to be Lipschitz continuous and defined everywhere, although the gradients of functions in $W^{1, \infty}(\Omega)$ can only be defined almost everywhere (denoted a.e.).

We call two matrices rank-one connected if their difference is a rank-one matrix. The classical Hadamard compatibility condition states that, given a plane with unit normal $n$ and two distinct constant matrices $F_{0}, F_{1} \in$ $\mathbb{R}^{3 \times 3}$, there exists a continuous deformation $y: \mathbb{R}^{3} \rightarrow \mathbb{R}^{3}$ such that $\nabla y$ takes the value $F_{0}$ on one side of the plane and $F_{1}$ on the other side if and only if $F_{0}$ and $F_{1}$ are rank-one connected as

$$
F_{1}-F_{0}=a \otimes n
$$

for some non-zero vector $a \in \mathbb{R}^{3}$. We next present a lemma that classifies all possible simple laminates formed by pairs of variants up to multiplication of rotations for the martensitic crystals in our discussion $[2,3,24]$. The lemma states that there is no rank-one connection between $\mathcal{U}_{i}$ and itself; and that for any $i, j \in K, i \neq j$, there are exactly two rank-one connections between $\mathcal{U}_{i}$ and $\mathcal{U}_{j}$.

Lemma 2.1. (1) For each $i \in K$, there do not exist matrices $R_{1} U_{i}$ and $R_{2} U_{i}$ with distinct $R_{1}, R_{2} \in S O(3)$ that are rank-one connected.

(2) For any $i, j \in K, i \neq j$, there are exactly two distinct $Q \in S O(3)$ such that

$$
Q U_{i}-U_{j}=a \otimes n
$$

for some $a, n \in \mathbb{R}^{3},|n|=1$, respectively.

We have that

$$
n \in\left\{ \pm e_{1}, \pm e_{2}\right\}
$$

for the orthorhombic to monoclinic transformation, and that

$$
n \in\left\{ \pm \frac{1}{\sqrt{2}}\left(e_{i}+e_{j}\right), \pm \frac{1}{\sqrt{2}}\left(e_{i}-e_{j}\right)\right\}
$$

for the cubic to tetragonal transformation.

We shall also assume that $F_{0}, F_{1} \in \mathcal{U}$ are rank-one connected as in (2.6), so

$$
(1-\lambda) F_{0}+\lambda F_{1}=F_{0}+\lambda a \otimes n, \quad \lambda \in \mathbb{R} .
$$

By Lemma 2.1, we can assume without loss of generality that $F_{1} \in \mathcal{U}_{1}$ and $F_{0} \in \mathcal{U}_{2}$, and we can also assume that

$$
n=e_{1}
$$

for the orthorhombic to monoclinic transformation and that

$$
n=\frac{1}{\sqrt{2}}\left(e_{1}+e_{2}\right)
$$


for the cubic to tetragonal transformation. We note that it can be shown under the hypotheses of Lemma 2.1 that if $\lambda \neq 0$ and $\lambda \neq 1$ then $[2,3,24]$

$$
(1-\lambda) F_{0}+\lambda F_{1}=F_{0}+\lambda a \otimes n \notin \mathcal{U} .
$$

The following lemma shows that any deformation with a gradient that is a mixture of the two matrices $F_{0}$ and $F_{1}$ must be a simple laminate.

Lemma 2.2. Let $y \in W^{1, \infty}\left(\Omega, \mathbb{R}^{3}\right)$ be such that

$$
\nabla y(x)=(1-\lambda(x)) F_{0}+\lambda(x) F_{1} \quad \text { a.e. } x \in \Omega,
$$

for the volume fraction $\lambda \in L^{\infty}(\Omega)$ satisfying $0 \leq \lambda(x) \leq 1$. We have that there exist unique $l \in W^{1, \infty}(\Omega)$ and $\hat{y} \in \mathbb{R}^{3}, \hat{y} \cdot a=0$, such that

$$
\begin{aligned}
y(x) & =F_{0} x+l(x) a+\hat{y}, & & x \in \Omega, \\
\nabla l(x) & =\lambda(x) n & & \text { a.e. } x \in \Omega .
\end{aligned}
$$

If $\tilde{\Omega} \subset \Omega$ is a subdomain with the property that $\{x \in \tilde{\Omega}: x \cdot n=\xi\}$ is connected for each $\xi \in \mathbb{R}$, then there exist $\tilde{l} \in W^{1, \infty}(\mathbb{R})$ and $\tilde{\lambda} \in L^{\infty}(\mathbb{R})$ such that

$$
\begin{array}{ll}
l(x)=\tilde{l}(x \cdot n), & x \in \tilde{\Omega}, \\
\lambda(x)=\tilde{\lambda}(x \cdot n) & \text { a.e. } x \in \tilde{\Omega}, \\
\tilde{l}^{\prime}(s)=\tilde{\lambda}(s) & \text { a.e. } s \in \mathbb{R} .
\end{array}
$$

Proof. The proof is identical to the proof of Proposition 1 in [2] for the case when $\lambda$ is a characteristic function. It follows by noting that if $w \in \mathbb{R}^{3}, w \cdot a=0$, then

$$
\nabla\left[\left(y(x)-F_{0} x\right) \cdot w\right]=0 \quad \text { a.e. } x \in \Omega .
$$

In this paper, we consider the minimization of the elastic energy (2.1) with respect to deformations $y \in W^{\phi}$ which are constrained by $y(x)=y_{\lambda}(x)$ for $x \in \partial \Omega$ where

$$
\begin{aligned}
& y_{\lambda}(x)=F_{0} x+l(x) a, \quad x \in \Omega, \\
& \nabla l(x)=\lambda(x) n \quad \text { a.e. } x \in \Omega,
\end{aligned}
$$

and where $l \in W^{1, \infty}(\Omega)$ and $\lambda \in L^{\infty}(\Omega)$ satisfies $0 \leq \lambda(x) \leq 1$. Our energy minimization problem is to minimize the energy (2.1) in the set of admissible deformations defined by

$$
W_{\lambda}^{\phi}=\left\{y \in W^{\phi}: y=y_{\lambda} \text { on } \partial \Omega\right\} .
$$

\section{Construction of EnERgy Minimizing SEQUenCeS}

We first consider the special case of $(2.8)$ where $\lambda(x)=\tilde{\lambda}(x \cdot n)$ for $\tilde{\lambda}(s) \in L^{\infty}(\mathbb{R})$, so

$$
y_{\lambda}(x)=F_{0} x+\left[\int_{0}^{x \cdot n} \tilde{\lambda}(s) d s+\zeta\right] a, \quad x \in \Omega,
$$

for some $\zeta \in \mathbb{R}$. 
We construct in two steps a family of deformations $\hat{u}_{\gamma} \in W^{1, \infty}\left(\mathbb{R}^{3} ; \mathbb{R}^{3}\right), \gamma \in\left(0, \gamma_{0}\right]$, for any fixed $\gamma_{0}>0$, satisfying

$$
\lim _{\gamma \rightarrow 0} \mathcal{E}\left(\hat{u}_{\gamma}\right)=0
$$

First, we construct $u_{\gamma} \in W^{1, \infty}\left(\mathbb{R}^{3} ; \mathbb{R}^{3}\right), \gamma \in\left(0, \gamma_{0}\right]$, which are simple laminates of scale $\gamma$ such that $\nabla u_{\gamma}(x)=F_{0}$ or $F_{1}$ for almost all $x \in \mathbb{R}^{3}$. Second, we construct $\hat{u}_{\gamma} \in W_{\lambda}^{\phi}, \gamma \in\left(0, \gamma_{0}\right]$, by modifying $u_{\gamma}$ by interpolation on the boundary.

Step 1. Construction of $u_{\gamma} \in W^{1, \infty}\left(\mathbb{R}^{3} ; \mathbb{R}^{3}\right), \gamma \in\left(0, \gamma_{0}\right]$. Set

$$
I_{\gamma}^{(i)}=((i-1) \gamma, i \gamma] \quad \text { and } \quad \lambda_{\gamma}^{(i)}=\frac{1}{\gamma} \int_{I_{\gamma}^{(i)}} \tilde{\lambda}(s) d s, \quad \forall i \in \mathbb{Z}
$$

Define the piecewise constant function $\tilde{\lambda}_{\gamma}: \mathbb{R} \rightarrow \mathbb{R}$ by

$$
\tilde{\lambda}_{\gamma}(s)=\lambda_{\gamma}^{(i)} \quad \text { if } s \in I_{\gamma}^{(i)}, i \in \mathbb{Z}
$$

and define the characteristic function $\chi_{\gamma}: \mathbb{R} \rightarrow \mathbb{R}$ by

$$
\chi_{\gamma}(s)=\left\{\begin{array}{lll}
1 & \text { if }(i-1) \gamma<s \leq\left(i-1+\lambda_{\gamma}^{(i)}\right) \gamma & \text { for some } i \in \mathbb{Z} \\
0 & \text { if }\left(i-1+\lambda_{\gamma}^{(i)}\right) \gamma<s \leq i \gamma & \text { for some } i \in \mathbb{Z}
\end{array}\right.
$$

Since $0 \leq \tilde{\lambda}(s), \tilde{\lambda}_{\gamma}(s), \chi_{\gamma}(s) \leq 1$ for almost all $s \in \mathbb{R}$, we have for any bounded interval $I \subset \mathbb{R}^{1}$ that

$$
\left|\int_{I}\left[\chi_{\gamma}(s)-\tilde{\lambda}(s)\right] d s\right| \leq 2 \gamma
$$

Define now

$$
u_{\gamma}(x)=F_{0} x+\left[\int_{0}^{x \cdot n} \chi_{\gamma}(s) d s+\zeta\right] a, \quad x \in \mathbb{R}^{3} .
$$

Obviously, $u_{\gamma} \in W^{1, \infty}\left(\mathbb{R}^{3} ; \mathbb{R}^{3}\right)$. Moreover, we have by (2.6) that

$$
\nabla u_{\gamma}(x)=F_{0}+\chi_{\gamma}(x \cdot n) a \otimes n \in\left\{F_{0}, F_{1}\right\} \quad \text { a.e. } x \in \mathbb{R}^{3} .
$$

In view of $(3.1,3.2)$, we also have

$$
\left|u_{\gamma}(x)-y_{\lambda}(x)\right| \leq 2|a| \gamma, \quad x \in \mathbb{R}^{3} .
$$

Step 2. Construction of $\hat{u}_{\gamma} \in W_{\lambda}^{\phi}, \gamma \in\left(0, \gamma_{0}\right]$. Set

$$
\Omega_{\gamma}=\{x \in \Omega: \operatorname{dist}(x, \partial \Omega)>v \gamma\}
$$

for some constant $v>0$ which will be specified later. Define $\psi_{\gamma}: \Omega \rightarrow \mathbb{R}$ by

$$
\psi_{\gamma}(x)= \begin{cases}1 & \text { if } x \in \Omega_{\gamma} \\ (v \gamma)^{-1} \operatorname{dist}(x, \partial \Omega) & \text { if } x \in \Omega \backslash \Omega_{\gamma}\end{cases}
$$


It is easy to see that $\psi_{\gamma} \in W^{1, \infty}(\Omega)$ and

$$
\begin{array}{ll}
0 \leq \psi_{\gamma}(x) \leq 1, & x \in \Omega, \\
\psi_{\gamma}(x)=1, & x \in \Omega_{\gamma}, \\
\psi_{\gamma}(x)=0, & x \in \partial \Omega, \\
\left|\nabla \psi_{\gamma}(x)\right| \leq(v \gamma)^{-1} & \text { a.e. } x \in \Omega .
\end{array}
$$

Now we define $\hat{u}_{\gamma}: \Omega \rightarrow \mathbb{R}^{3}$ for $\gamma \in\left(0, \gamma_{0}\right]$ by

$$
\hat{u}_{\gamma}(x)=\psi_{\gamma}(x) u_{\gamma}(x)+\left(1-\psi_{\gamma}(x)\right) y_{\lambda}(x), \quad x \in \Omega .
$$

It is easy to verify that

$$
\nabla \hat{u}_{\gamma}(x)=\left[u_{\gamma}(x)-y_{\lambda}(x)\right] \otimes \nabla \psi_{\gamma}(x)+\psi_{\gamma}(x) \nabla u_{\gamma}(x)+\left(1-\psi_{\gamma}(x)\right) \nabla y_{\lambda}(x)
$$

for almost all $x \in \Omega$. By (3.3) - (3.6), we have for all $\gamma \in\left(0, \gamma_{0}\right]$ that

$$
\left\|\nabla \hat{u}_{\gamma}(x)\right\| \leq C \quad \text { a.e. } x \in \Omega,
$$

where $C$ here and below is a constant independent of $\gamma$, and that

$$
\nabla \hat{u}_{\gamma}(x) \in\left\{F_{0}, F_{1}\right\} \quad \text { a.e. } x \in \Omega_{\gamma} .
$$

Therefore, $\hat{u}_{\gamma} \in W_{\lambda}^{\phi}$ for any $\gamma \in\left(0, \gamma_{0}\right]$ by the continuity of the energy density $\phi$. Moreover, $\operatorname{meas}\left(\Omega \backslash \Omega_{\gamma}\right)=\mathrm{O}(\gamma)$ as $\gamma \rightarrow 0$ since $\Omega$ is a Lipschitz domain, so by (3.7), (3.8), and (2.2) we have

$$
\mathcal{E}\left(\hat{u}_{\gamma}\right)=\mathrm{O}(\gamma) \quad \text { as } \gamma \rightarrow 0 .
$$

By the rank-one connection (2.6), we have that

$$
\operatorname{det} F_{1}=\operatorname{det}\left(F_{0}+a \otimes n\right)=\left(\operatorname{det} F_{0}\right)\left(1+F_{0}^{-1} a \cdot n\right) .
$$

This together with the fact that $\operatorname{det} F_{0}=\operatorname{det} F_{1}>0$ (see (2.4) and (2.5)) implies that

$$
F_{0}^{-1} a \cdot n=0 .
$$

Consequently, for any $\xi \in \mathbb{R}$, we have

$$
\operatorname{det}\left(F_{0}+\xi a \otimes n\right)=\left(\operatorname{det} F_{0}\right)\left(1+\xi F_{0}^{-1} a \cdot n\right)=\operatorname{det} F_{0} .
$$

It now follows from the equations (2.7) and (3.3) that

$$
\psi_{\gamma}(x) \nabla u_{\gamma}(x)+\left(1-\psi_{\gamma}(x)\right) \nabla y_{\lambda}(x)=F_{0}+\xi(x) a \otimes n \quad \text { a.e. } x \in \Omega,
$$

where

$$
\xi(x)=\psi_{\gamma}(x) \chi_{\gamma}(x \cdot n)+\left(1-\psi_{\gamma}(x)\right) \lambda(x) \quad \text { a.e. } x \in \Omega .
$$

Thus,

$$
\operatorname{det}\left[\psi_{\gamma}(x) \nabla u_{\gamma}(x)+\left(1-\psi_{\gamma}(x)\right) \nabla y_{\lambda}(x)\right]=\operatorname{det} F_{0}=\operatorname{det} F_{1}>0 \quad \text { a.e. } x \in \Omega \text {. }
$$


Choosing $v>0$ large enough, we can therefore conclude from (3.4) - (3.6) that

$$
\operatorname{det} \nabla \hat{u}_{\gamma}(x) \geq C>0 \quad \text { a.e. } x \in \Omega, \forall \gamma \in\left(0, \gamma_{0}\right] .
$$

We summarize our results in the following theorem.

Theorem 3.1. If $y_{\lambda}(x)$ has the form (3.1), then there exist a family of deformations $\hat{u}_{\gamma} \in W_{\lambda}^{\phi}, \gamma \in\left(0, \gamma_{0}\right]$, for any fixed $\gamma_{0}>0$, such that (3.9) holds and such that

$$
\lim _{\gamma \rightarrow 0} \mathcal{E}\left(\hat{u}_{\gamma}\right)=0
$$

Theorem 3.1 can be directly extended to more general deformations $y_{\lambda}(x)$ such as described in the following corollary.

Corollary 3.1. We suppose that $\Omega_{i} \subset \Omega$ for $i=1, \ldots, M$ are disjoint Lipschitz subdomains such that

$$
\bar{\Omega}=\bigcup_{i=1}^{M} \bar{\Omega}_{i}
$$

and we also suppose that there exist $\tilde{\lambda}_{i} \in L^{\infty}(\mathbb{R})$ for $i=1, \ldots, M$ such that

$$
\lambda(x)=\tilde{\lambda}_{i}(x \cdot n) \quad \text { a.e. } x \in \Omega_{i} .
$$

It then follows that there exist a family of deformations $\hat{u}_{\gamma} \in W_{\lambda}^{\phi}, \gamma \in\left(0, \gamma_{0}\right]$, for any fixed $\gamma_{0}>0$, such that (3.9) holds and such that

$$
\lim _{\gamma \rightarrow 0} \mathcal{E}\left(\hat{u}_{\gamma}\right)=0
$$

Proof. We construct deformations $\hat{u}_{i \gamma}$ defined on $\bar{\Omega}_{i}$ for $i=1, \ldots, M$ such that

$$
\hat{u}_{i \gamma}(x)=y_{\lambda}(x), \quad x \in \partial \Omega_{i},
$$

by the technique of Theorem 3.1 applied to $\Omega_{i}$, and we then construct $\hat{u}_{\gamma} \in W_{\lambda}^{\phi}$ by

$$
\hat{u}_{\gamma}(x)=\hat{u}_{i \gamma}(x), \quad x \in \Omega_{i}, i=1, \ldots, M .
$$

\section{Approximation of the Limiting macroscopic Deformation}

In this section and in the next section, we assume only that $y_{\lambda}(x)$ is of the general form (2.8). Our first lemma below follows immediately from the growth rate of the energy density around the energy wells (2.3).

Lemma 4.1. We have

$$
\int_{\Omega}\|\nabla y(x)-\pi(\nabla y(x))\|^{2} d x \leq \kappa^{-1} \mathcal{E}(y), \quad \forall y \in W^{\phi} .
$$

Notice that by the above lemma we have that $W^{\phi} \subset W^{1,2}\left(\Omega, \mathbb{R}^{3}\right)$. In what follows we shall denote by $C$ a generic positive constant which will be independent of all $y \in W_{\lambda}^{\phi}$. 
Lemma 4.2. There exists a constant $C>0$ such that

$$
\int_{\Omega}\left|\left[\pi(\nabla y(x))-\nabla y_{\lambda}(x)\right] w\right|^{2} d x \leq C \mathcal{E}(y)^{\frac{1}{2}}, \quad \forall y \in W_{\lambda}^{\phi},
$$

for all $w \in \mathbb{R}^{3}$ satisfying $w \cdot n=0$ and $|w|=1$.

Proof. We first prove the lemma for the orthorhombic to monoclinic transformation. We have that

$$
\pi(F) \in \mathrm{SO}(3) F_{0} \cup \mathrm{SO}(3) F_{1}, \quad \forall F \in \mathbb{R}^{3 \times 3} .
$$

Fix $w \in \mathbb{R}^{3}$ with $w \cdot n=0$ and $|w|=1$. By (2.6) and (2.7), we have that

$$
\nabla y_{\lambda}(x) w=F_{0} w=F_{1} w \quad \text { a.e. } x \in \Omega,
$$

leading to

$$
|\pi(F) w|=\left|\nabla y_{\lambda}(x) w\right| \quad \text { a.e. } x \in \Omega, \forall F \in \mathbb{R}^{3 \times 3} .
$$

Fix $y \in W_{\lambda}^{\phi}$. Since $y(x)=y_{\lambda}(x)$ on $\partial \Omega$, we have by the divergence theorem that

$$
\int_{\Omega} \nabla y(x) d x=\int_{\Omega} \nabla y_{\lambda}(x) d x .
$$

It follows from (4.1-4.3), the Cauchy-Schwarz inequality, and Lemma 4.1 that

$$
\begin{aligned}
\int_{\Omega} \mid[\pi(\nabla y(x))- & \left.\nabla y_{\lambda}(x)\right]\left.w\right|^{2} d x=2 \int_{\Omega} \nabla y_{\lambda}(x) w \cdot\left[\nabla y_{\lambda}(x)-\pi(\nabla y(x))\right] w d x \\
& =2 F_{1} w \cdot \int_{\Omega}[\nabla y(x)-\pi(\nabla y(x))] w d x \\
& \leq 2\left|F_{1} w\right|(\operatorname{meas} \Omega)^{\frac{1}{2}}\left[\int_{\Omega}\|\nabla y(x)-\pi(\nabla y(x))\|^{2} d x\right]^{\frac{1}{2}} \\
& \leq C \mathcal{E}(y)^{\frac{1}{2}}
\end{aligned}
$$

Next, we prove the lemma for the cubic to tetragonal transformation. Recall that in this case the normal $n$ is given as $n=\left(e_{1}+e_{2}\right) / \sqrt{2}$. Set

$$
w_{1}=\frac{1}{\sqrt{3}}\left(e_{1}-e_{2}+e_{3}\right) \quad \text { and } \quad w_{2}=\frac{1}{\sqrt{3}}\left(e_{1}-e_{2}-e_{3}\right) .
$$

We can easily verify that

$$
w_{1} \cdot n=w_{2} \cdot n=0, \quad\left|w_{1}\right|=\left|w_{2}\right|=1,
$$

and

$$
\left|U_{i} w_{j}\right|=\sqrt{\frac{2 \eta_{1}^{2}+\eta_{2}^{2}}{3}}, \quad i=1,2,3, j=1,2 .
$$

We can thus conclude by (4.1) that (4.2), hence (4.4), also holds true for $w=w_{1}$ and $w=w_{2}$, respectively. We have in fact proved the desired inequality in this case as well, since $\left\{w_{1}, w_{2}\right\}$ is a basis for the two-dimensional subspace $\left\{w \in \mathbb{R}^{3}: w \cdot n=0\right\}$. 
We next give an error bound for the $L^{2}$ approximation of the directional derivative of the limiting macroscopic deformation $y_{\lambda}$ in any direction tangential to parallel layers of the laminate. It is a direct consequence of the triangle inequality and the above two lemmas.

Theorem 4.1. There exists a constant $C>0$ such that

$$
\int_{\Omega}\left|\left[\nabla y(x)-\nabla y_{\lambda}(x)\right] w\right|^{2} d x \leq C\left[\mathcal{E}(y)^{\frac{1}{2}}+\mathcal{E}(y)\right], \quad \forall y \in W_{\lambda}^{\phi},
$$

for all $w \in \mathbb{R}^{3}$ satisfying $w \cdot n=0$ and $|w|=1$.

We now give an error bound for the $L^{2}$ approximation of the limiting macroscopic deformation $y_{\lambda}$ by the admissible deformations $y \in W_{\lambda}^{\phi}$.

Theorem 4.2. There exists a constant $C>0$ such that

$$
\int_{\Omega}\left|y(x)-y_{\lambda}(x)\right|^{2} d x \leq C\left[\mathcal{E}(y)^{\frac{1}{2}}+\mathcal{E}(y)\right], \quad \forall y \in W_{\lambda}^{\phi} .
$$

Proof. Let $z \in C^{1}\left(\bar{\Omega} ; \mathbb{R}^{3}\right)$ and $w \in \mathbb{R}^{3}$ with $|w|=1$. It follows from the Poincaré inequality $[25,34]$ that

$$
\int_{\Omega}|z(x)|^{2} d x \leq C\left[\int_{\partial \Omega}|z(x)|^{2} d S+\int_{\Omega}|\nabla z(x) w|^{2} d x\right],
$$

where $C=C(\Omega)$ is a positive constant independent of $z$. This inequality is also true for any $z \in W^{\phi}$ by the density of $C^{1}\left(\bar{\Omega} ; \mathbb{R}^{3}\right)$ in $W^{\phi}$. Setting $z=y-y_{\lambda}$ for any $y \in W_{\lambda}^{\phi}$, we obtain the desired result by Theorem 4.1 with $w \in \mathbb{R}^{3}$ so chosen that $w \cdot n=0$ and $|w|=1$.

The next corollary states that the infimum of the energy is not generally attained on $W_{\lambda}^{\phi}$.

Corollary 4.1. There does not exist $y \in W_{\lambda}^{\phi}$ such that $\mathcal{E}(y)=0$ if

$$
\operatorname{meas}\{x \in \Omega: 0<\lambda(x)<1\}>0 .
$$

Proof. We assume that there exist $y \in W_{\lambda}^{\phi}$ such that $\mathcal{E}(y)=0$. By Theorem 4.2, we have that $y=y_{\lambda}$. It follows from (4.6) that there is an integer $p \geq 3$ such that the set

$$
\omega_{p}=\left\{x \in \Omega: \frac{1}{p} \leq \lambda(x) \leq 1-\frac{1}{p}\right\}
$$

has positive measure. On the other hand, the set

$$
\Delta_{p}=\left\{\left(1-\lambda_{0}\right) F_{0}+\lambda_{0} F_{1} \in \mathbb{R}^{3 \times 3}: \frac{1}{p} \leq \lambda_{0} \leq 1-\frac{1}{p}\right\}
$$

is compact in $\mathbb{R}^{3 \times 3}$ and is disjoint with $\mathcal{U}$ by Lemma 2.1. Consequently, the continuous energy density $\phi$ reaches its minimum $m\left(\Delta_{p}\right)>0$ on the set $\Delta_{p}$. We obtain a contradiction since

$$
0=\mathcal{E}(y)=\mathcal{E}\left(y_{\lambda}\right) \geq \int_{\omega_{p}} \phi\left(\nabla y_{\lambda}(x)\right) d x \geq m\left(\Delta_{p}\right) \text { meas } \omega_{p}>0
$$


Now we establish an error bound for the weak $L^{2}$ approximation of the limiting macroscopic deformation gradient $\nabla y_{\lambda}$. It follows from such an error bound that for any energy minimizing sequence $\left\{y_{k}\right\}_{k=1}^{\infty}$ the corresponding sequence of gradients $\left\{\nabla y_{k}\right\}_{k=1}^{\infty}$ converges weakly to the deformation gradient $\nabla y_{\lambda}$.

Theorem 4.3. For any Lipschitz domain $\omega \subset \Omega$, there exists a constant $C=C(\omega)>0$ such that

$$
\left\|\int_{\omega}\left[\nabla y(x)-\nabla y_{\lambda}(x)\right] d x\right\| \leq C\left[\mathcal{E}(y)^{\frac{1}{8}}+\mathcal{E}(y)^{\frac{1}{2}}\right], \quad \forall y \in W_{\lambda}^{\phi} .
$$

Proof. We have from the divergence theorem and the Cauchy-Schwarz inequality that

$$
\begin{gathered}
\left\|\int_{\omega}\left[\nabla y(x)-\nabla y_{\lambda}(x)\right] d x\right\|=\left\|\int_{\partial \omega}\left[y(x)-y_{\lambda}(x)\right] \otimes \nu d S\right\| \\
\leq \int_{\partial \omega}\left|y(x)-y_{\lambda}(x)\right| d S \leq\left(\operatorname{meas}_{2} \partial \omega\right)^{\frac{1}{2}}\left(\int_{\partial \omega}\left|y(x)-y_{\lambda}(x)\right|^{2} d S\right)^{\frac{1}{2}}
\end{gathered}
$$

for any $y \in W_{\lambda}^{\phi}$ where $\nu$ is the unit exterior normal to $\partial \omega$ and meas $2 \partial \omega$ is the surface area of $\partial \omega$. By the trace theorem [1] we have

$$
\begin{aligned}
& \int_{\partial \omega}\left|y(x)-y_{\lambda}(x)\right|^{2} d S \leq C\left[\int_{\omega}\left|y(x)-y_{\lambda}(x)\right|^{2} d x+\int_{\omega}|\nabla| y(x)-\left.\nabla y_{\lambda}(x)\right|^{2} \mid d x\right] \\
& \leq C\left[\int_{\omega}\left|y(x)-y_{\lambda}(x)\right|^{2} d x+\int_{\omega}\left|y(x)-y_{\lambda}(x)\right|\left\|\nabla\left[y(x)-y_{\lambda}(x)\right]\right\| d x\right] \\
& \leq C\left[\int_{\Omega}\left|y(x)-y_{\lambda}(x)\right|^{2} d x+\left(\int_{\Omega}\left|y(x)-y_{\lambda}(x)\right|^{2} d x\right)^{\frac{1}{2}}\left(\int_{\Omega}\left\|\nabla y(x)-\nabla y_{\lambda}(x)\right\|^{2} d x\right)^{\frac{1}{2}}\right] .
\end{aligned}
$$

We also have by the triangle inequality and Lemma 4.1 that

$$
\begin{aligned}
\left(\int_{\Omega}\left\|\nabla y(x)-\nabla y_{\lambda}(x)\right\|^{2} d x\right)^{\frac{1}{2}} & \leq\left(\int_{\Omega}\|\nabla y(x)-\pi(\nabla y(x))\|^{2} d x\right)^{\frac{1}{2}}+\left(\int_{\Omega}\left\|\pi(\nabla y(x))-\nabla y_{\lambda}(x)\right\|^{2} d x\right)^{\frac{1}{2}} \\
& \leq C\left[\mathcal{E}(y)^{\frac{1}{2}}+1\right] .
\end{aligned}
$$

Hence, we obtain from using the inequality of Theorem 4.2 and (4.9) in (4.8) that

$$
\int_{\partial \omega}\left|y(x)-y_{\lambda}(x)\right|^{2} d S \leq C\left[\mathcal{E}(y)^{\frac{1}{4}}+\mathcal{E}(y)\right]
$$

which, together with (4.7), leads to the desired inequality.

\section{Approximation of the simple laminate}

We define the projection operator $\pi_{12}: \mathbb{R}^{3 \times 3} \rightarrow \mathcal{U}_{1} \cup \mathcal{U}_{2}$ by

$$
\left\|F-\pi_{12}(F)\right\|=\min _{G \in \mathcal{U}_{1} \cup \mathcal{U}_{2}}\|F-G\|, \quad \forall F \in \mathbb{R}^{3 \times 3} .
$$

We note that $\pi_{12}=\pi$ for the orthorhombic to monoclinic transformation. Next, we define the operators $\Theta: \mathbb{R}^{3 \times 3} \rightarrow \mathrm{SO}(3)$ and $\Pi: \mathbb{R}^{3 \times 3} \rightarrow\left\{F_{0}, F_{1}\right\}$ by the identity

$$
\pi_{12}(F)=\Theta(F) \Pi(F), \quad \forall F \in \mathbb{R}^{3 \times 3} .
$$


The following lemma reduces the three-well problem for the cubic to tetragonal transformation to a two-well problem. Its proof indicates that the measure of the set of points at which the deformation gradient for an energy minimizing sequence is near $\mathcal{U}_{3}$ converges to zero.

Lemma 5.1. For the cubic to tetragonal transformation, there exists a constant $C>0$ such that

$$
\int_{\Omega}\left\|\nabla y(x)-\pi_{12}(\nabla y(x))\right\|^{2} d x \leq C\left[\mathcal{E}(y)^{\frac{1}{2}}+\mathcal{E}(y)\right], \quad \forall y \in W_{\lambda}^{\phi} .
$$

Proof. It is easy to check that

$$
\inf _{F \in \mathcal{U}_{3}}\left|\left[F-\nabla y_{\lambda}(x)\right] e_{3}\right| \geq\left|\eta_{2}-\eta_{1}\right| \quad \text { a.e. } x \in \Omega
$$

For a fixed $y \in W_{\lambda}^{\phi}$, we set

$$
\Omega_{3}=\left\{x \in \Omega: \pi(\nabla y(x)) \in \mathcal{U}_{3}\right\} .
$$

Since $e_{3} \cdot n=0$, it follows from Lemma 4.2 that

$$
\text { meas } \Omega_{3}=\int_{\Omega_{3}} d x \leq\left|\eta_{2}-\eta_{1}\right|^{-2} \int_{\Omega_{3}}\left|\left[\pi(\nabla y(x))-\nabla y_{\lambda}(x)\right] e_{3}\right|^{2} d x \leq C \mathcal{E}(y)^{\frac{1}{2}} .
$$

Consequently, we have by Lemma 4.1 that

$$
\begin{aligned}
\int_{\Omega}\left\|\nabla y(x)-\pi_{12}(\nabla y(x))\right\|^{2} d x & \leq 2 \int_{\Omega}\|\nabla y(x)-\pi(\nabla y(x))\|^{2} d x+2 \int_{\Omega}\left\|\pi(\nabla y(x))-\pi_{12}(\nabla y(x))\right\|^{2} d x \\
& \leq 2 \int_{\Omega}\|\nabla y(x)-\pi(\nabla y(x))\|^{2} d x+8\left(2 \eta_{1}^{2}+\eta_{2}^{2}\right) \text { meas } \Omega_{3} \\
& \leq C\left[\mathcal{E}(y)^{\frac{1}{2}}+\mathcal{E}(y)\right],
\end{aligned}
$$

completing the proof.

We now give an error bound for the projection operator $\Pi: \mathbb{R}^{3 \times 3} \rightarrow\left\{F_{0}, F_{1}\right\}$.

Theorem 5.1. There exists a constant $C>0$ such that

$$
\int_{\Omega}\|\nabla y(x)-\Pi(\nabla y(x))\|^{2} d x \leq C\left[\mathcal{E}(y)^{\frac{1}{2}}+\mathcal{E}(y)\right], \quad \forall y \in W_{\lambda}^{\phi} .
$$

Proof. We have for any $w \in \mathbb{R}^{3}$ such that $w \cdot n=0$ the identity

$$
\Pi(F) w=F_{0} w=F_{1} w=\nabla y_{\lambda}(x) w \quad \text { a.e. } x \in \Omega, \forall F \in \mathbb{R}^{3 \times 3} .
$$

Hence, we obtain from (5.1) that

$$
\begin{aligned}
{[\Theta(F)-I] F_{0} w } & =[\Theta(F)-I] \Pi(F) w=\left[\pi_{12}(F)-\nabla y_{\lambda}(x)\right] w \\
& =\left[\pi_{12}(F)-\pi(F)\right] w+\left[\pi(F)-\nabla y_{\lambda}(x)\right] w \quad \text { a.e. } x \in \Omega, \forall F \in \mathbb{R}^{3 \times 3} .
\end{aligned}
$$

We substitute $F=\nabla y(x)$ for any $y \in W_{\lambda}^{\phi}$ and $x \in \Omega$ in the above identity, and estimate the two terms by (5.2) 
and Lemma 4.2 to obtain by the triangle inequality that

$$
\begin{aligned}
\int_{\Omega}\left|[\Theta(\nabla y(x))-I] F_{0} w\right|^{2} d x & \leq 2 \int_{\Omega}\left|\left[\pi_{12}(\nabla y(x))-\pi(\nabla y(x))\right] w\right|^{2} d x+2 \int_{\Omega}\left|\left[\pi(\nabla y(x))-\nabla y_{\lambda}(x)\right] w\right|^{2} d x \\
& \leq C \mathcal{E}(y)^{\frac{1}{2}}
\end{aligned}
$$

We next fix $w_{1} \in \mathbb{R}^{3}$ and $w_{2} \in \mathbb{R}^{3}$ so that $w_{1} \cdot n=w_{2} \cdot n=0$ and that $w_{1}, w_{2}$ are linearly independent. We have for $m=F_{0} w_{1} \times F_{0} w_{2}$ that

$$
Q m=Q F_{0} w_{1} \times Q F_{0} w_{2}, \quad \forall Q \in \mathrm{SO}(3) .
$$

Thus, it follows that for all $F \in \mathbb{R}^{3 \times 3}$ we have

$$
\begin{aligned}
{[\Theta(F)-I] m } & =\left\{\Theta(F) F_{0} w_{1} \times \Theta(F) F_{0} w_{2}\right\}-\left\{F_{0} w_{1} \times F_{0} w_{2}\right\} \\
& =\left\{[\Theta(F)-I] F_{0} w_{1} \times \Theta(F) F_{0} w_{2}\right\}-\left\{F_{0} w_{1} \times[I-\Theta(F)] F_{0} w_{2}\right\} .
\end{aligned}
$$

We obtain from the above inequality and (5.3) that

$$
\int_{\Omega}|[\Theta(\nabla y(x))-I] m|^{2} d x \leq C \mathcal{E}(y)^{\frac{1}{2}} .
$$

Since $\left\{F_{0} w_{1}, F_{0} w_{2}, m\right\}$ is a basis for $\mathbb{R}^{3}$, it follows from (5.3) and (5.4) that

$$
\int_{\Omega}\|[\Theta(\nabla y(x))-I]\|^{2} d x \leq C \mathcal{E}(y)^{\frac{1}{2}} .
$$

The proof is completed by applying the triangle inequality to the identity

$$
\begin{aligned}
F-\Pi(F) & =\left[F-\pi_{12}(F)\right]+\left[\pi_{12}(F)-\Pi(F)\right] \\
& =\left[F-\pi_{12}(F)\right]+[\Theta(F)-I] \Pi(F), \quad \forall F \in \mathbb{R}^{3 \times 3},
\end{aligned}
$$

with $F=\nabla y(x)$ for $x \in \Omega$, and by estimating the two terms by Lemma 5.1 and (5.5).

The following theorem gives an estimate for the approximation of volume fractions. It states that for any energy minimizing sequence $\left\{y_{k}\right\}$ in $W_{\lambda}^{\phi}$ the volume fraction that $\nabla y_{k}(x)$ is near $F_{0}$ converges to $1-\lambda(x)$ and the volume fraction that $\nabla y_{k}(x)$ is near $F_{1}$ converges to $\lambda(x)$. The statement of the theorem utilizes the subsets

$$
\begin{aligned}
& \omega_{\rho}^{0}(y)=\left\{x \in \omega: \Pi(\nabla y(x))=F_{0} \text { and }\left\|F_{0}-\nabla y(x)\right\|<\rho\right\}, \\
& \omega_{\rho}^{1}(y)=\left\{x \in \omega: \Pi(\nabla y(x))=F_{1} \text { and }\left\|F_{1}-\nabla y(x)\right\|<\rho\right\},
\end{aligned}
$$

which are defined for any subset $\omega \subset \Omega, \rho>0$, and $y \in W_{\lambda}^{\phi}$. We also denote the mean value of $\lambda$ on $\omega$ by

$$
\hat{\lambda}_{\omega}=\frac{1}{\operatorname{meas} \omega} \int_{\omega} \lambda(x) d x
$$

Theorem 5.2. For any Lipschitz domain $\omega \subset \Omega$ and any $\rho>0$ there exists a positive constant $C$ such that

$$
\operatorname{meas}\left(\omega-\left\{\omega_{\rho}^{0}(y) \cup \omega_{\rho}^{1}(y)\right\}\right) \leq C\left[\mathcal{E}(y)^{\frac{1}{2}}+\mathcal{E}(y)\right], \quad \forall y \in W_{\lambda}^{\phi},
$$


and

$$
\left|\frac{\operatorname{meas} \omega_{\rho}^{0}(y)}{\operatorname{meas} \omega}-\left(1-\hat{\lambda}_{\omega}\right)\right|+\left|\frac{\operatorname{meas} \omega_{\rho}^{1}(y)}{\operatorname{meas} \omega}-\hat{\lambda}_{\omega}\right| \leq C\left[\mathcal{E}(y)^{\frac{1}{8}}+\mathcal{E}(y)\right], \quad \forall y \in W_{\lambda}^{\phi}
$$

Proof. We shall assume that $y \in W_{\lambda}^{\phi}$. We have by the definition of $\omega_{\rho}^{0} \equiv \omega_{\rho}^{0}(y)$ and $\omega_{\rho}^{1} \equiv \omega_{\rho}^{1}(y)$ that

$$
\begin{aligned}
\operatorname{meas}\left(\omega-\left\{\omega_{\rho}^{0} \cup \omega_{\rho}^{1}\right\}\right) & \leq \frac{1}{\rho} \int_{\omega-\left\{\omega_{\rho}^{0} \cup \omega_{\rho}^{1}\right\}}\|\Pi(\nabla y(x))-\nabla y(x)\| d x \\
& \leq \frac{\left[\operatorname{meas}\left(\omega-\left\{\omega_{\rho}^{0} \cup \omega_{\rho}^{1}\right\}\right)\right]^{\frac{1}{2}}}{\rho}\left[\int_{\Omega}\|\Pi(\nabla y(x))-\nabla y(x)\|^{2} d x\right]^{\frac{1}{2}} .
\end{aligned}
$$

Consequently, we have

$$
\operatorname{meas}\left(\omega-\left\{\omega_{\rho}^{0} \cup \omega_{\rho}^{1}\right\}\right) \leq \frac{1}{\rho^{2}}\left[\int_{\Omega}\|\Pi(\nabla y(x))-\nabla y(x)\|^{2} d x\right]
$$

which together with Theorem 5.1 implies (5.6).

We have by (2.7) that

$$
\begin{aligned}
{\left[\operatorname{meas} \omega_{\rho}^{0}-\left(1-\hat{\lambda}_{\omega}\right) \text { meas } \omega\right] F_{0}+\left[\operatorname{meas} \omega_{\rho}^{1}-\right.} & \left.\hat{\lambda}_{\omega} \text { meas } \omega\right] F_{1} \\
& =\int_{\omega}\left[\Pi(\nabla y(x))-\nabla y_{\lambda}(x)\right] d x-\int_{\omega-\left\{\omega_{\rho}^{0} \cup \omega_{\rho}^{1}\right\}} \Pi(\nabla y(x)) d x .
\end{aligned}
$$

By the triangle inequality, the Cauchy-Schwarz inequality, Theorem 5.1, and Theorem 4.3, we have that

$$
\begin{aligned}
\left\|\int_{\omega}\left[\Pi(\nabla y(x))-\nabla y_{\lambda}(x)\right] d x\right\| & \leq\left\|\int_{\omega}[\Pi(\nabla y(x))-\nabla y(x)] d x\right\|+\left\|\int_{\omega}\left[\nabla y(x)-\nabla y_{\lambda}(x)\right] d x\right\| \\
& \leq(\operatorname{meas} \omega)^{\frac{1}{2}}\left[\int_{\Omega}\|\Pi(\nabla y(x))-\nabla y(x)\|^{2} d x\right]^{\frac{1}{2}}+\left\|\int_{\omega}\left[\nabla y(x)-\nabla y_{\lambda}(x)\right] d x\right\| \\
& \leq C\left[\mathcal{E}(y)^{\frac{1}{8}}+\mathcal{E}(y)^{\frac{1}{2}}\right] .
\end{aligned}
$$

We also have by (5.6) that

$$
\left\|\int_{\omega-\left\{\omega_{\rho}^{0} \cup \omega_{\rho}^{1}\right\}} \Pi(\nabla y(x)) d x\right\| \leq C \operatorname{meas}\left(\omega-\left\{\omega_{\rho}^{0} \cup \omega_{\rho}^{1}\right\}\right) \leq C\left[\mathcal{E}(y)^{\frac{1}{2}}+\mathcal{E}(y)\right] .
$$

Finally, the inequality (5.7) follows from (5.8)-(5.10) and the linear independence of $F_{0}$ and $F_{1}$.

The final theorem in this section gives an estimate for nonlinear functions of the deformation gradient. The estimate utilizes the Sobolev space $\mathcal{V}$ of all functions $f \in L^{2}\left(\Omega \times \mathbb{R}^{3 \times 3}\right)$ such that

$$
\|f\|_{\mathcal{V}}^{2}=\int_{\Omega}\left[\underset{F \in \mathbb{R}^{3 \times 3}}{\operatorname{ess} \sup _{F}}\left\|\nabla_{F} f(x, F)\right\|\right]^{2} d x+\left\|G_{f}\right\|_{W^{1,2}(\Omega)}^{2}<\infty,
$$


where

$$
G_{f}(x)=f\left(x, F_{1}\right)-f\left(x, F_{0}\right), \quad x \in \Omega .
$$

Functions in the space $\mathcal{V}$ can represent thermodynamic variables of the underlying crystal.

Theorem 5.3. There exists a constant $C>0$ such that

$$
\begin{aligned}
\left|\int_{\Omega}\left\{f(x, \nabla y(x))-\left[(1-\lambda(x)) f\left(x, F_{0}\right)+\lambda(x) f\left(x, F_{1}\right)\right]\right\} d x\right| \\
\leq C\|f\|_{\mathcal{V}}\left[\mathcal{E}(y)^{\frac{1}{4}}+\mathcal{E}(y)^{\frac{1}{2}}\right], \quad \forall y \in W_{\lambda}^{\phi}, \forall f \in \mathcal{V} .
\end{aligned}
$$

Proof. We have the decomposition

$$
\begin{aligned}
\int_{\Omega}\{f & \left.(x, \nabla y(x))-\left[(1-\lambda(x)) f\left(x, F_{0}\right)+\lambda(x) f\left(x, F_{1}\right)\right]\right\} d x \\
= & \int_{\Omega}[f(x, \nabla y(x))-f(x, \Pi(\nabla y(x)))] d x \\
& \quad+\int_{\Omega}\left\{f(x, \Pi(\nabla y(x)))-\left[(1-\lambda(x)) f\left(x, F_{0}\right)+\lambda(x) f\left(x, F_{1}\right)\right]\right\} d x \\
= & \mathcal{J}_{1}+\mathcal{J}_{2} .
\end{aligned}
$$

The first term $\mathcal{J}_{1}$ can be estimated by Theorem 5.1 as follows:

$$
\begin{aligned}
\left|\mathcal{J}_{1}\right| & \leq \int_{\Omega}\left[\underset{F \in \mathbb{R}^{3 \times 3}}{\operatorname{ess} \sup }\left\|\nabla_{F} f(x, F)\right\|\right]\|\nabla y(x)-\Pi(\nabla y(x))\| d x \\
& \leq\left\{\int_{\Omega}\left[\operatorname{esssup}_{F \in \mathbb{R}^{3 \times 3}}\left\|\nabla_{F} f(x, F)\right\|\right]^{2} d x\right\}^{\frac{1}{2}}\left[\int_{\Omega}\|\nabla y(x)-\Pi(\nabla y(x))\|^{2} d x\right]^{\frac{1}{2}} \\
& \leq C\|f\|_{\mathcal{V}}\left[\mathcal{E}(y)^{\frac{1}{4}}+\mathcal{E}(y)^{\frac{1}{2}}\right] .
\end{aligned}
$$

By (2.6) and the definition of $\Pi: \mathbb{R}^{3 \times 3} \rightarrow\left\{F_{0}, F_{1}\right\}$, we have the identity

$$
f(x, \Pi(F))-\left[(1-\lambda(x)) f\left(x, F_{0}\right)+\lambda(x) f\left(x, F_{1}\right)\right]=\frac{1}{|a|^{2}}\left\{a \cdot\left[\Pi(F)-\nabla y_{\lambda}(x)\right] n\right\} G_{f}(x),
$$

for all $F \in \mathbb{R}^{3 \times 3}$ and for almost all $x \in \Omega$, leading to

$$
\begin{aligned}
\mathcal{J}_{2} & =\int_{\Omega}\left\{f(x, \Pi(\nabla y(x)))-\left[(1-\lambda(x)) f\left(x, F_{0}\right)+\lambda(x) f\left(x, F_{1}\right)\right]\right\} d x \\
& =\frac{1}{|a|^{2}} \int_{\Omega}\left\{a \cdot\left[\Pi(\nabla y(x))-\nabla y_{\lambda}(x)\right] n\right\} G_{f}(x) d x \\
& =\frac{1}{|a|^{2}} \int_{\Omega}\{a \cdot[\Pi(\nabla y(x))-\nabla y(x)] n\} G_{f}(x) d x+\frac{1}{|a|^{2}} \int_{\Omega}\left\{a \cdot\left[\nabla y(x)-\nabla y_{\lambda}(x)\right] n\right\} G_{f}(x) d x \\
& =\frac{1}{|a|^{2}} \int_{\Omega}\{a \cdot[\Pi(\nabla y(x))-\nabla y(x)] n\} G_{f}(x) d x-\frac{1}{|a|^{2}} \int_{\Omega}\left\{a \cdot\left[y(x)-y_{\lambda}(x)\right]\right\}\left\{\nabla G_{f}(x) \cdot n\right\} d x,
\end{aligned}
$$


where we used the divergence theorem and the fact that $y(x)=y_{\lambda}(x)$ for any $y \in W_{\lambda}^{\phi}$ and for all $x \in \partial \Omega$. It next follows from the Cauchy-Schwarz inequality, Theorem 4.2, and Theorem 5.1 that

$$
\begin{aligned}
\left|\mathcal{J}_{2}\right| & \leq C\left\{\int_{\Omega}\left[\left|\nabla G_{f}(x) \cdot n\right|^{2}+G_{f}(x)^{2}\right] d x\right\}^{\frac{1}{2}}\left[\mathcal{E}(y)^{\frac{1}{4}}+\mathcal{E}(y)^{\frac{1}{2}}\right] \\
& \leq C\|f\|_{\mathcal{V}}\left[\mathcal{E}(y)^{\frac{1}{4}}+\mathcal{E}(y)^{\frac{1}{2}}\right] .
\end{aligned}
$$

We finally obtain the inequality (5.11) from (5.12-5.14).

\section{Finite element approximations}

For simplicity, we shall assume in what follows that the reference configuration $\Omega \subset \mathbb{R}^{3}$ is a polyhedral domain. For a fixed positive number $h_{0}$, let $\tau_{h}, 0<h \leq h_{0}$, be a family of tetrahedral finite element meshes of $\Omega$, such that $\bar{\Omega}=\cup_{T \in \tau_{h}} T$, where $h$ is the maximum diameter of any tetrahedron $T$ in the mesh $\tau_{h}$. We shall assume as usual that any face of any tetrahedron in a mesh $\tau_{h}$ has a disjoint interior with respect to any other tetrahedron in that mesh and that any face of a tetrahedron is either a subset of the boundary $\partial \Omega$ or is the face of another tetrahedron in the mesh $\tau_{h}$. Let $\mathcal{A}_{h}, 0<h \leq h_{0}$, be the corresponding family of piecewise linear, continuous finite element spaces with respect to the mesh $\tau_{h}[11,30]$.

We can define the interpolation operator $\mathcal{I}_{h}: C\left(\bar{\Omega} ; \mathbb{R}^{3}\right) \rightarrow \mathcal{A}_{h}$ for each $h \in\left(0, h_{0}\right]$ which interpolates the values at the vertices of the tetrahedral elements $T$ of $\tau_{h}$. We will assume that the family $\tau_{h}$ of finite element meshes is quasi-regular $[11,30]$, so that

$$
\underset{x \in \Omega}{\operatorname{ess} \sup }\left\|\nabla \mathcal{I}_{h} y(x)\right\| \leq C \underset{x \in \Omega}{\operatorname{ess} \sup }\|\nabla y(x)\|
$$

for all $y \in W^{1, \infty}\left(\Omega ; \mathbb{R}^{3}\right)$, where the constant $C$ in (6.1) and below will always denote a generic positive constant independent of $h$. We also note for $y \in C\left(\bar{\Omega} ; \mathbb{R}^{3}\right)$ that

$$
\left.\mathcal{I}_{h} y(x)\right|_{T}=\left.y(x)\right|_{T} \text { for any } T \in \tau_{h} \text { such that }\left.y(x)\right|_{T} \in\left\{P^{1}(T)\right\}^{3},
$$

for $h \in\left(0, h_{0}\right]$, where $\left\{P^{1}(T)\right\}^{3} \equiv P^{1}(T) \times P^{1}(T) \times P^{1}(T)$ and $P^{1}(T)$ denotes the space of linear polynomials defined on $T$.

Since $\Omega$ is the union of disjoint tetrahedra, we can assume in this section by Lemma 2.2 that there exist disjoint Lipschitz subdomains $\Omega_{i} \subset \Omega$ for $i=1, \ldots, M$ with $\bar{\Omega}=\bigcup_{i=1}^{M} \bar{\Omega}_{i}, \tilde{l}_{i} \in W^{1, \infty}(\mathbb{R}), \tilde{\lambda}_{i} \in L^{\infty}(\mathbb{R})$, and $\hat{y}_{i} \in \mathbb{R}^{3}, \hat{y}_{i} \cdot a=0$, such that

$$
\begin{aligned}
y_{\lambda}(x) & =F_{0} x+\tilde{l}_{i}(x \cdot n) a+\hat{y}_{i}, & & x \in \Omega_{i}, \\
\tilde{l}_{i}^{\prime}(s) & =\lambda_{i}(s), & & \text { a.e. } s \in \mathbb{R} .
\end{aligned}
$$

Recall from Section 2 that since $y_{\lambda} \in W^{1, \infty}\left(\Omega ; \mathbb{R}^{3}\right), y_{\lambda}$ can be uniquely represented as a Lipschitz continuous function defined on $\bar{\Omega}$. So, we can then uniquely define the finite element deformation $y_{\lambda h} \in \mathcal{A}_{h}$ by

$$
y_{\lambda h}=\mathcal{I}_{h} y_{\lambda}(x), \quad x \in \bar{\Omega},
$$

and we can define the finite element space of admissible deformations

$$
\mathcal{A}_{\lambda h}=\left\{y_{h} \in \mathcal{A}_{h}: y_{h}(x)=y_{\lambda h}(x) \text { on } x \in \partial \Omega\right\} .
$$


We also note that the trace $\left.y_{\lambda}\right|_{\partial \Omega} \in W^{1, \infty}\left(\partial \Omega ; \mathbb{R}^{3}\right)$ is then well-defined, and it follows from well-known estimates for the interpolation error $[11,30]$ that

$$
\left\|y_{\lambda}-y_{\lambda h}\right\|_{L^{\infty}\left(\partial \Omega ; \mathbf{R}^{3}\right)} \leq C h\left\|y_{\lambda}\right\|_{W^{1, \infty}\left(\partial \Omega ; \mathbf{R}^{3}\right)} .
$$

In what follows we shall use the result that $y_{\lambda h} \in \mathcal{A}_{\lambda h}, 0<h \leq h_{0}$, satisfies the condition

$$
\left\|y_{\lambda}-y_{\lambda h}\right\|_{L^{2}\left(\partial \Omega ; \mathbf{R}^{3}\right)} \leq C h .
$$

We begin our analysis of the finite element approximation of a laminate with varying volume fractions with the following result on the minimization of the energy $\mathcal{E}$ on the space $\mathcal{A}_{\lambda h}$.

Theorem 6.1. There exists $y_{h} \in \mathcal{A}_{\lambda h}$ for each $h \in\left(0, h_{0}\right]$ such that

$$
\mathcal{E}\left(y_{h}\right)=\min _{z_{h} \in \mathcal{A}_{\lambda h}} \mathcal{E}\left(z_{h}\right) \leq C h^{1 / 2} .
$$

Proof. The existence of $y_{h} \in \mathcal{A}_{\lambda h}$ can be proven by the same argument as in the proof of Theorem 6.1 in [22]. To prove the inequality in (6.3) we follow the argument given in [24] to show that $\hat{y}_{h}=\mathcal{I}_{h} \hat{u}_{\gamma} \in \mathcal{A}_{\lambda h}$ with $\hat{u}_{\gamma}(x)$ defined by Corollary 3.1 and $\gamma=h^{1 / 2}$ satisfies

$$
\mathcal{E}\left(\hat{y}_{h}\right) \leq C h^{1 / 2} .
$$

We next give a series of estimates for the finite element approximation of the deformation $y_{\lambda}$ by deformations $y_{h} \in \mathcal{A}_{\lambda h}$. These estimates follow those for the deformations $y \in W_{\lambda}^{\phi}$ given in previous sections.

Theorem 6.2. We have for any $w \in \mathbb{R}^{3}$ such that $w \cdot n=0$ and $|w|=1$ that

$$
\int_{\Omega}\left|\left[\nabla y_{h}(x)-\nabla y_{\lambda}(x)\right] w\right|^{2} d x \leq C\left[\mathcal{E}\left(y_{h}\right)^{\frac{1}{2}}+\mathcal{E}\left(y_{h}\right)+\left\|y_{\lambda}-y_{\lambda h}\right\|_{L^{2}\left(\partial \Omega ; \mathbb{R}^{3}\right)}\right], \quad \forall y_{h} \in \mathcal{A}_{\lambda h} .
$$

Proof. Fix $y_{h} \in \mathcal{A}_{\lambda h}$ and $w \in \mathbb{R}^{3}$ such that $w \cdot n=0$ and $|w|=1$. By the decomposition

$$
y_{h}-y_{\lambda}=\left[y_{h}-\pi\left(y_{h}\right)\right]+\left[\pi\left(y_{h}\right)-y_{\lambda}\right]
$$

and Lemma 4.1, we need only to prove

$$
\int_{\Omega}\left|\left[\pi\left(\nabla y_{h}(x)\right)-\nabla y_{\lambda}(x)\right] w\right|^{2} d x \leq C\left[\mathcal{E}\left(y_{h}\right)^{\frac{1}{2}}+\left\|y_{\lambda}-y_{\lambda h}\right\|_{L^{2}\left(\partial \Omega ; \mathbb{R}^{3}\right)}\right] .
$$

We only consider the orthorhombic to monoclinic transformation, since the cubic to tetragonal transformation can be treated similarly (see the proofs of Lemma 4.2 and Theorem 4.1). Noting that $y_{h}(x)=y_{\lambda h}(x)$ for $x \in \partial \Omega$, we have by (4.1) and the divergence theorem that

$$
\begin{aligned}
\int_{\Omega}\left|\left[\pi\left(\nabla y_{h}(x)\right)-\nabla y_{\lambda}(x)\right] w\right|^{2} d x & =2 F_{0} w \cdot \int_{\Omega}\left[\nabla y_{\lambda}(x)-\pi\left(\nabla y_{h}(x)\right)\right] w d x \\
& =2 F_{0} w \cdot\left\{\int_{\Omega}\left[\nabla y_{\lambda}(x)-\nabla y_{h}(x)\right] d x+\int_{\Omega}\left[\nabla y_{h}(x)-\pi\left(\nabla y_{h}(x)\right)\right] d x\right\} w \\
& =2 F_{0} w \cdot\left\{\int_{\partial \Omega}\left[y_{\lambda}(x)-y_{\lambda h}(x)\right] \otimes \nu d S+\int_{\Omega}\left[\nabla y_{h}(x)-\pi\left(\nabla y_{h}(x)\right)\right] d x\right\} w
\end{aligned}
$$


This, together with Lemma 4.1 and the Cauchy-Schwarz inequality, leads to (6.4).

Theorem 6.3. We have

$$
\int_{\Omega}\left|y_{h}(x)-y_{\lambda}(x)\right|^{2} d x \leq C\left[\mathcal{E}\left(y_{h}\right)^{\frac{1}{2}}+\mathcal{E}\left(y_{h}\right)+\left\|y_{\lambda}-y_{\lambda h}\right\|_{L^{2}\left(\partial \Omega ; \mathbb{R}^{3}\right)}+\left\|y_{\lambda}-y_{\lambda h}\right\|_{L^{2}\left(\partial \Omega ; \mathbb{R}^{3}\right)}^{2}\right], \forall y_{h} \in \mathcal{A}_{\lambda h}
$$

Proof. Fix $y_{h} \in \mathcal{A}_{h}$. Setting $z=y_{h}-y_{\lambda}$ and choosing $w \in \mathbb{R}^{3}$ so that $w \cdot n=1$ and $|w|=1$, we obtain the desired inequality by (4.5) and Theorem 6.2.

By an argument similar to the proof of Theorem 4.3, we can use the above theorem to obtain the following result on the weak convergence of finite element approximations.

Theorem 6.4. For any Lipschitz domain $\omega \subset \Omega$ we have that

$$
\left\|\int_{\omega}\left[\nabla y_{h}(x)-\nabla y_{\lambda}(x)\right] d x\right\| \leq C\left[\mathcal{E}\left(y_{h}\right)^{\frac{1}{8}}+\mathcal{E}\left(y_{h}\right)\right]+C\left[\left\|y_{\lambda}-y_{\lambda h}\right\|_{L^{2}\left(\partial \Omega ; \mathbb{R}^{3}\right)}^{\frac{1}{4}}+\left\|y_{\lambda}-y_{\lambda h}\right\|_{L^{2}\left(\partial \Omega ; \mathbb{R}^{3}\right)}\right], \forall y_{h} \in \mathcal{A}_{\lambda h}
$$

Recall the operator $\Pi: \mathbb{R}^{3 \times 3} \rightarrow\left\{F_{0}, F_{1}\right\}$ defined by (5.1). We have the following result which is parallel to Theorem 5.1. The key estimate is (6.4).

Theorem 6.5. We have

$$
\int_{\Omega}\left\|\nabla y_{h}(x)-\Pi\left(\nabla y_{h}(x)\right)\right\|^{2} d x \leq C\left[\mathcal{E}\left(y_{h}\right)^{\frac{1}{2}}+\mathcal{E}\left(y_{h}\right)+\left\|y_{\lambda}-y_{\lambda h}\right\|_{L^{2}\left(\partial \Omega ; \mathbb{R}^{3}\right)}\right], \quad \forall y_{h} \in \mathcal{A}_{\lambda h}
$$

Recall that $\hat{\lambda}_{\omega}$ is the average of $\lambda$ on $\omega$. Using the same argument as in the proof of Theorem 5.2 , we can obtain the following result from Theorem 6.4 and Theorem 6.5.

Theorem 6.6. For any Lipschitz domain $\omega \subset \Omega$ and any $\rho>0$ we have that

$$
\operatorname{meas}\left(\omega-\left\{\omega_{\rho}^{0}\left(y_{h}\right) \cup \omega_{\rho}^{1}\left(y_{h}\right)\right\}\right) \leq C\left[\mathcal{E}\left(y_{h}\right)^{\frac{1}{2}}+\mathcal{E}\left(y_{h}\right)+\left\|y_{\lambda}-y_{\lambda h}\right\|_{L^{2}\left(\partial \Omega ; \mathbb{R}^{3}\right)}\right], \quad \forall y_{h} \in \mathcal{A}_{\lambda h}
$$

and

$$
\begin{aligned}
& \left|\frac{\operatorname{meas} \omega_{\rho}^{0}\left(y_{h}\right)}{\operatorname{meas} \omega}-\left(1-\hat{\lambda}_{\omega}\right)\right|+\left|\frac{\operatorname{meas} \omega_{\rho}^{1}\left(y_{h}\right)}{\operatorname{meas} \omega}-\hat{\lambda}_{\omega}\right| \\
& \quad \leq C\left[\mathcal{E}\left(y_{h}\right)^{\frac{1}{8}}+\mathcal{E}\left(y_{h}\right)+\left\|y_{\lambda}-y_{\lambda h}\right\|_{L^{2}\left(\partial \Omega ; \mathbb{R}^{3}\right)}^{\frac{1}{4}}+\left\|y_{\lambda}-y_{\lambda h}\right\|_{L^{2}\left(\partial \Omega ; \mathbb{R}^{3}\right)}\right], \quad \forall y_{h} \in \mathcal{A}_{\lambda h} .
\end{aligned}
$$

By slightly modifying the proof of Theorem 5.3, we can obtain the following result corresponding to Theorem 5.3 for admissible finite element deformations.

Theorem 6.7. We have

$$
\begin{aligned}
& \left|\int_{\Omega}\left\{f\left(x, \nabla y_{h}(x)\right)-\left[(1-\lambda(x)) f\left(x, F_{0}\right)+\lambda(x) f\left(x, F_{1}\right)\right]\right\} d x\right| \\
& \quad \leq C\|f\|_{\mathcal{V}}\left[\mathcal{E}\left(y_{h}\right)^{\frac{1}{4}}+\mathcal{E}\left(y_{h}\right)^{\frac{1}{2}}+\left\|y_{\lambda}-y_{\lambda h}\right\|_{L^{2}\left(\partial \Omega ; \mathbb{R}^{3}\right)}^{\frac{1}{2}}+\left\|y_{\lambda}-y_{\lambda h}\right\|_{L^{2}\left(\partial \Omega ; \mathbb{R}^{3}\right)}\right]
\end{aligned}
$$

for all $y_{h} \in \mathcal{A}_{\lambda h}$ and all $f \in \mathcal{V}$. 
Optimization techniques applied to the problem

$$
\inf _{y_{h} \in \mathcal{A}_{\lambda h}} \mathcal{E}\left(y_{h}\right)
$$

compute one of the many local minima given by approximations on different length scales to the same optimal microstructure [24]. For this reason, we give error estimates for finite element deformations $y_{h} \in \mathcal{A}_{\lambda h}$ that satisfy the following quasi-optimality condition

$$
\mathcal{E}\left(y_{h}\right) \leq \alpha \inf _{z_{h} \in \mathcal{A}_{\lambda h}} \mathcal{E}\left(z_{h}\right)
$$

for some constant $\alpha \geq 1$ independent of $h$.

It follows directly from the previous theorems in this section and (6.2) that we can obtain the following error estimates for quasi-optimal finite element deformations $y_{h} \in \mathcal{A}_{\lambda h}$.

Corollary 6.1. We have

$$
\int_{\Omega}\left|\left[\nabla y_{h}(x)-\nabla y_{\lambda}(x)\right] w\right|^{2} d x \leq C h^{\frac{1}{4}}
$$

for any $w \in \mathbb{R}^{3}$ such that $w \cdot n=1$ and $|w|=1$ and for any $y_{h} \in \mathcal{A}_{\lambda h}$ which satisfies the quasi-optimality condition (6.6).

Corollary 6.2. We have

$$
\int_{\Omega}\left|y_{h}(x)-y_{\lambda}(x)\right|^{2} d x \leq C h^{\frac{1}{4}}
$$

for any $y_{h} \in \mathcal{A}_{\lambda h}$ which satisfies the quasi-optimality condition (6.6).

Corollary 6.3. If $\omega \subset \Omega$ is a Lipschitz domain, then there exists a positive constant $C$, independent of $h$, such that

$$
\left\|\int_{\omega}\left[\nabla y_{h}(x)-\nabla y_{\lambda}(x)\right] d x\right\| \leq C h^{\frac{1}{16}}
$$

for any $y_{h} \in \mathcal{A}_{\lambda h}$ which satisfies the quasi-optimality condition (6.6).

Corollary 6.4. We have

$$
\int_{\Omega}\left\|\nabla y_{h}(x)-\Pi\left(\nabla y_{h}(x)\right)\right\|^{2} d x \leq C h^{\frac{1}{4}}
$$

for any $y_{h} \in \mathcal{A}_{\lambda h}$ which satisfies the quasi-optimality condition (6.6).

Corollary 6.5. For any Lipschitz domain $\omega \subset \Omega$ and any $\rho>0$ we have

$$
\operatorname{meas}\left(\omega-\left\{\omega_{\rho}^{0}\left(y_{h}\right) \cup \omega_{\rho}^{1}\left(y_{h}\right)\right\}\right) \leq C h^{\frac{1}{4}}
$$


and

$$
\left|\frac{\operatorname{meas} \omega_{\rho}^{0}\left(y_{h}\right)}{\operatorname{meas} \omega}-\left(1-\hat{\lambda}_{\omega}\right)\right|+\left|\frac{\operatorname{meas} \omega_{\rho}^{1}\left(y_{h}\right)}{\operatorname{meas} \omega}-\hat{\lambda}_{\omega}\right| \leq C h^{\frac{1}{16}}
$$

for any $y_{h} \in \mathcal{A}_{\lambda h}$ which satisfies the quasi-optimality condition (6.6).

Corollary 6.6. We have

$$
\left|\int_{\Omega}\left\{f\left(x, \nabla y_{h}(x)\right)-\left[(1-\lambda(x)) f\left(x, F_{0}\right)+\lambda(x) f\left(x, F_{1}\right)\right]\right\} d x\right| \leq C\|f\|_{\mathcal{V}} h^{\frac{1}{8}}
$$

for any $f \in \mathcal{V}$ and any $y_{h} \in \mathcal{A}_{\lambda h}$ which satisfies the quasi-optimality condition (6.6).

\section{REFERENCES}

[1] R. Adams, Sobolev Spaces. Academic Press, New York (1975).

[2] J. Ball and R. James, Fine phase mixtures as minimizers of energy. Arch. Rat. Mech. Anal. 100 (1987) 13-52.

[3] J. Ball and R. James, Proposed experimental tests of a theory of fine microstructure and the two-well problem. Phil. Trans. R. Soc. Lond. A 338 (1992) 389-450.

[4] Z.S. Basinski and J.W. Christian, Experiments on the martensitic transformation in single crystals of indium-thallium alloys. Acta Metall. 2 (1954) 148-166.

[5] M.W. Burkart and T.A. Read, Diffusionless phase changes in the indium-thallium system. Trans. AIME J. Metals 197 (1953) 1516-1524.

[6] C. Carstensen and P. Plecháč, Numerical solution of the scalar double-well problem allowing microstructure. Math. Comp. 66 (1997) 997-1026.

[7] M. Chipot, Numerical analysis of oscillations in nonconvex problems. Numer. Math. 59 (1991) 747-767.

[8] M. Chipot and C. Collins, Numerical approximations in variational problems with potential wells. SIAM J. Numer. Anal. 29 (1992) 1002-1019.

[9] M. Chipot, C. Collins and D. Kinderlehrer, Numerical analysis of oscillations in multiple well problems. Numer. Math. 70 (1995) 259-282.

[10] M. Chipot and D. Kinderlehrer, Equilibrium configurations of crystals. Arch. Rat. Mech. Anal. 103 (1988) $237-277$.

[11] P. Ciarlet, The Finite Element Method for Elliptic Problems. North-Holland, Amsterdam (1978).

[12] C. Collins, D. Kinderlehrer and M. Luskin, Numerical approximation of the solution of a variational problem with a double well potential. SIAM J. Numer. Anal. 28 (1991) 321-332.

[13] C. Collins and M. Luskin, Optimal order estimates for the finite element approximation of the solution of a nonconvex variational problem. Math. Comp. 57 (1991) 621-637.

[14] J. Ericksen, Constitutive theory for some constrained elastic crystals. J. Solids and Structures 22 (1986) 951-964.

[15] J. Ericksen, Stable equilibrium configurations of elastic crystals. Arch. Rat. Mech. Anal. (1986) 1-14.

[16] D. French, On the convergence of finite element approximations of a relaxed variational problem. SIAM J. Numer. Anal. 28, (1991) 419-436.

[17] P.-A. Gremaud, Numerical analysis of a nonconvex variational problem related to solid-solid phase transitions. SIAM J. Numer. Anal. 31 (1994) 111-127.

[18] R. James, Minimizing sequences and the microstructure of crystals in Proceedings of the Society of Metals Conference on Phase Transformations. Cambridge University Press (1989).

[19] D. Kinderlehrer, Twinning in crystals II. In Metastability and Incompletely Posed Problems. S. Antman, J.L. Ericksen, D. Kinderlehrer and I. Muller Eds. IMA Volumes in Mathematics and Its Applications. Springer-Verlag, New York 3 (1987) 185-212.

[20] D. Kinderlehrer and P. Pedregal, Characterizations of gradient Young measures. Arch. Rat. Mech. Anal. 115, (1991) 329-365.

[21] R. Kohn, Relaxation of a double-well energy. Cont. Mech. Thermodyn. 3 (1991) 193-236.

[22] B. Li and M. Luskin, Finite element analysis of microstructure for the cubic to tetragonal transformation. SIAM J. Numer. Anal. 35 (1998) 376-392. 
[23] B. Li and M. Luskin, Nonconforming finite element approximation of crystalline microstructure. Math. Comp. 67 (1998) 917-946.

[24] M. Luskin, On the computation of crystalline microstructure. Acta Numerica (1996) 191-257.

[25] M. Luskin, Approximation of a laminated microstructure for a rotationally invariant, double well energy density. Numer. Math. 75 (1997) 205-221.

[26] M. Luskin and L. Ma, Analysis of the finite element approximation of microstructure in micromagnetics. SIAM J. Numer. Anal. 29 (1992) 320-331.

[27] R.A. Nicolaides and N. Walkington, Strong convergence of numerical solutions to degenerate variational problems. Math. Comp. 64 (1995) 117-127.

[28] P. Pedregal, Numerical approximation of parametrized measures. Num. Funct. Anal. Opt. 16, (1995) 1049-1066.

[29] P. Pedregal, On the numerical analysis of non-convex variational problems. Numer. Math. 74 (1996) 325-336.

[30] A. Quarteroni and A. Valli, Numerical Approximation of Partial Differential Equations. Springer-Verlag, Berlin (1994).

[31] T. Roubíček, Numerical approximation of relaxed variational problems. J. Convex Analysis 3, (1996) 329-347.

[32] V. Šverák, Lower-semicontinuity of variational integrals and compensated compactness in Proceedings ICM 94 Birkhäuser. Zürich (1995)

[33] L. Tartar, Compensated compactness and applications to partial differential equations in Nonlinear analysis and mechanics. Pitman Research Notes in Mathematics. R. Knops Eds. Pitman, London (1978)

[34] J. Wloka, Partial Differential Equations. Cambridge University Press, Cambridge (1987). 